\title{
Spectrum and Antibiotic Resistance Pattern of Uropathogens Causing Urinary Tract Infection Among Inpatients and Outpatients: An Experience of a Tertiary Care Hospital in Karachi, Pakistan
}

\author{
Farheen Mubashir ${ }^{1 *}$, Madiha Sattar ${ }^{2}$, Farhan Essa ${ }^{1}$ and Saleem Hafiz ${ }^{3}$ \\ ${ }^{1}$ Department of Microbiology and Infection Control, Essa Laboratory, Karachi, Pakistan \\ ${ }^{2}$ Department of Microbiology and Infection Control, Dr. Ruth K. M. Pafu Civil Hospital, Karachi, Pakistan \\ ${ }^{3}$ Department of Microbiology, SIUT, Karachi, Pakistan
}

\begin{abstract}
Background: UTI leads to irrational use of antibiotics and may cause higher antibiotic resistance which is a major public health issue. Therefore, it is crucial for clinicians to acquire knowledge of etiological agents and susceptibility patterns in their population for the optimistic use of antibiotic medicines.

Objective: To determine the spectrum and antibiotic resistance pattern of uropathogens causing urinary tract infection among inpatients and outpatients in a tertiary care hospital in Karachi.

Methods: This descriptive cross-sectional study was conducted in the Department of Microbiology, Sindh Institute of Urology and Transplant, Karachi, The study was conducted from March 2016 to March 2017 after taking approval from the Hospital Ethics Committee. Urine specimens were analyzed to establish a diagnosis of UTI and identify uropahtogens. The antibiotic susceptibility pattern of uropathogens was studied using disc diffusion method against the following antibiotics; fosfomycin, ampicillin, amoxicillin-clavulanate, nitrofurantoin, cefotaxime, ceftazidime, amikacin, cefoxitin, imipenem and vancomycin.
\end{abstract}

Results: A total of 480 samples of UTI were received during the study period. The average age of patients was $54.79 \pm 12.09$ years. The majority of samples came out positive from the male gender $(65 \%)$ and in-patient department $(n=400,83.3 \%)$. The highest prevalent microorganism was E.coli (82.1\%) followed by Klebsiella spp (14\%), Pseudomonas aeruginosa (1\%), Proteus mirabilis (1\%), Morganella morgannii (1\%) and Staphylococcus aureus (0.8\%). All microorganisms were highly resistant to augmentin, cefoxitin, cefotaxime, ceftazidime. Only pseudomonas aeruginosa was highly resistant to imipenem (60\%). Pseudomonas aeruginosa (100\%), E.coli (86.8\%) and Klebsiella spp (71.6\%) were highly sensitive for Amikacin (100\%). Morganella morgannii (80\%) and Proteus Mirabilis (40\%) were mainly resistant to Fosfomycin. Only E.coli was sensitive to nitrofurantoin $(74.1 \%)$.

Conclusion: The present study demonstrated that gram-negative bacteria was the most frequent cause of urinary tract infection. Microorganisms showed variable resistance to different antibiotics. The first line of antibiotics should be rationally selected by physicians to treat urinary tract infections.

Keywords: Urinary tract infections, antimicrobial drugs, antibiotic resistance, gram-positive bacteria, gram-negative bacteria.

\section{INTRODUCTION}

Urinary tract infection (UTI) is termed as a health condition that is associated with clinical signs and detection of pathogens in the kidney, urine, urethra, prostate and bladder [1]. Globally, UTI ranks as the second most frequently occurring bacterial infection that affects individuals of different age groups. Globally, UTI accounts for 150-250 million cases in a year and is the most frequent reason for a patient to seek medical advice [2, 3].

Nearly $40 \%$ to $50 \%$ of women and $5 \%$ of men develop UTI at least once in their life [4-6]. Both the gender are affected by UTI regardless of their age but the high prevalence among females may be due to anatomical structures due to anatomical structures or high bacterial load in urothelial mucosa or other factors such as obstructed urinary tract, sexual activity and pregnancy [7].

*Corresponding author: Farheen Mubashir, Department of Microbiology, Essa Laboratory, Karachi, Pakistan;

Email:farheenmubashir@yahoo.com

Received: April 14, 2021; Revised: May 18, 2021; Accepted: May 19, 2021 DOI: https://doi.org/10.37184/lnjpc.2707-3521.3.2
Gram-positive and gram-negative bacteria are involved in the etiology of UTI but, uropathogenic Escherichia coli (E.coli) is a cause of the majority of cases UTI among the general population [8, 9]. Other most probably uropathogens involved in UTI are Klebsiella, Enterobacter, Serratia, Proteus, Pseudomonas, and Enterococcus, Staphylococcus aureus [10]. A study showed a predominance of E.coli in $31.5 \%$ of UTI patients whereas the prevalence of other pathogens among the general population was $3.5 \%$ [11].

There is evidence to conclude that UTI is associated with huge consumption of antibiotics in and out of the hospital and irrational use of antibiotics may cause higher antibiotic resistance which is a major public health issue around the world [12]. It is crucial for clinicians to acquire knowledge of etiological agents and susceptibility patterns in their population for the optimistic use of antibiotic medicines. The susceptibility pattern of uropathogens varies with time and geographical region, different settings thus, each 
institute should monitor the drug sensitivity pattern in their settings to identify changes causing agents and their susceptibility pattern for appropriate management of the patient. Therefore, we planned to conduct the current study to determine the spectrum and antibiotic resistance pattern of uropathogens causing urinary tract infection among inpatients and outpatients in tertiary care hospitals in Karachi.

\section{METHODOLOGY}

The current descriptive cross-sectional study was conducted at the Department of Microbiology at Sindh Institute of Urology and Transplantation from March 2016 to March 2017. Patients of both the gender of age range 18 to 60 years diagnosed with UTI for at least 2 months ago and showing the presence of single organism with pure growth more than 104 organisms on CLED plate were included in the study. Patients of a mixed growth of multiple organisms or single organism but with growth less than 104 organisms on CLED plate and taking antibiotics in the past 14 days were excluded from the study. The prevalence of pathogens other than E.coli in the general population was 3.5\% [11]. Therefore, the sample size of 450 was calculated taking a prevalence of $3.5 \%$ at a $95 \%$ confidence interval and a margin of error of $1.7 \%$ using World Health Organization (WHO) calculator version 2.1.

The urine specimen was taken from the patient and sent to the Microbiology department for analysis. The final diagnosis of UTI was established with detection of positive culture in urine specimen which was defined as $>10^{4}$ colony-forming units per milliliter from voided specimens or 50,000 colony-forming units per milliliter from a catheterized specimen. The volume of $0.1 \mathrm{ml}$ of urine was inoculated on a cystine lactose electrolyte deficient medium plate (CLED) (Oxoid, UK) to isolate uropathogens which was incubated at $37^{\circ} \mathrm{C}$ for 24 hours. Inoculation of urine was done with the help of a sterilized calibrated wire loop and the number of colonies was counted on each plate. Significant bacterial growth was considered for colony count $>105$ organisms $/ \mathrm{mL}$ of urine [13]. International guidelines were used to biochemically characterize the colonies [14]. MacConkey agar (Oxoid, UK) was used to subculture the isolated which was kept for incubation at $37^{\circ} \mathrm{C}$ for 24 hours to achieve pure growth.

The antimicrobial susceptibility pattern of uropathogens was evaluated using Kirby-Bauer disk diffusion. The inhibition zone of bacterial growth was interpreted according to Clinical and Laboratory Standard guidelines [15]. Isolates showing intermediate susceptibility were defined as resistant. Escherichia coli American Type
Culture Collection (ATCC) 25922, Pseudomonas aeruginosa 27853 and Staphylococcus aureus 29213 were used as quality control strains. All gram-positive and gram-negative isolates were tested for the following antibiotics; fosfomycin, ampicillin, amoxicillin-clavulanate, nitrofurantoin, cefotaxime, ceftazidime, amikacin, cefoxitin, imipenem and vancomycin. Statistical package SPSS version 21 was used for data entry and analysis. Variables were summarized as frequencies with percentages and mean \pm standard deviation for categorical and numerical variables respectively.

\section{RESULTS}

During the study period, a total of 480 samples were found UTI positive with a mean age of $54.79 \pm 12.09$ years. Most of specimen came out to be positives from males $(n=312,65 \%)$ and in-patient department $(n=400$, $83.3 \%$ ). The majority of the detected isolates were gram-negative bacteria $(n=476,99.2 \%)$. Out of 480 samples, the most frequently detected organism was E.coli $(\mathrm{n}=394,82.1 \%)$ followed by Klebsiella spp $(\mathrm{n}=67$, $14 \%)$, Pseudomonas aeruginosa $(n=5,1 \%)$, Proteus mirabilis $(n=5,1 \%)$, Morganella morgannii $(n=5,1 \%)$ and Staphylococcus aureus ( $n=4,0.8 \%)$. Gender-wise frequency of isolates is presented in Table 1.

The resistance pattern of isolates against the tested antibiotics is depicted in Table 2. All microorganisms were highly resistant to augmentin, cefoxitin, cefotaxime, ceftazidime. Only pseudomonas aeruginosa was highly resistant to imipenem (60\%). Pseudomonas aeruginosa (100\%), E.coli (86.8\%) and Klebsiella spp (71.6) were highly sensitive for Amikacin (100\%). Morganella morgannii $(80 \%)$ and Proteus mirabilis (40\%) were mainly resistant to Fosfomycin. Only E.coli was sensitive to nitrofurantoin (74.1\%).

Table 1: Frequency of isolated microorganisms among males and females.

\begin{tabular}{l|c|c}
\hline Microorganism & $\begin{array}{c}\text { Males } \\
\mathbf{n}(\%)\end{array}$ & $\begin{array}{c}\text { Females } \\
\mathbf{n}(\%)\end{array}$ \\
\hline Gram-negative organisms & $255(64.7)$ & $139(35.3)$ \\
\hline Escherichia coli & $40(59.7)$ & $27(40.3)$ \\
\hline klebsiella spp & $4(80)$ & $1(20)$ \\
\hline Pseudomonas aeruginosa & $5(100)$ & $0(0)$ \\
\hline Proteus mirabilis & $5(100)$ & $0(0)$ \\
\hline Morganella morganii & $3(75)$ & $1(25)$ \\
\hline Gram-positive organisms & \multicolumn{1}{|l}{} \\
\hline Staphylococcus aureus &
\end{tabular}


Table 2: Resistance pattern of isolates against the tested antibiotics.

\begin{tabular}{l|c|c|c|c|c|c}
\hline Drugs & $\begin{array}{c}\text { Escherichia coli } \\
\mathbf{n}(\%)\end{array}$ & $\begin{array}{c}\text { klebsiella spp } \\
\mathbf{n}(\%)\end{array}$ & $\begin{array}{c}\text { Pseudomonas } \\
\text { aeruginosa } \\
\mathbf{n}(\%)\end{array}$ & $\begin{array}{c}\text { Proteus mirabilis } \\
\mathbf{n}(\%)\end{array}$ & $\begin{array}{c}\text { Morganella } \\
\text { morganii } \\
\mathbf{n}(\%)\end{array}$ & $\begin{array}{c}\text { Staphylococcus } \\
\text { aureus } \\
\mathbf{n}(\%)\end{array}$ \\
\hline Augmentin & $249(63.2)$ & $37(55.2 \%)$ & $3(60 \%)$ & $5(100 \%)$ & $5(100 \%)$ & $4(100 \%)$ \\
\hline Cefoxitin & $276(70.1 \%)$ & $49(73.1 \%)$ & $5(100 \%)$ & $3(60 \%)$ & $3(60 \%)$ & $4(100 \%)$ \\
\hline Imipenem & $29(7.4 \%)$ & $9(13.4 \%)$ & $3(60 \%)$ & $0(0 \%)$ & $1(20 \%)$ & $1(25 \%)$ \\
\hline Amikacin & $52(13.2 \%)$ & $19(28.4 \%)$ & $0(0 \%)$ & $2(40 \%)$ & $2(40 \%)$ & $2(50 \%)$ \\
\hline Fostomycin & $35(8.9 \%)$ & $12(17.9 \%)$ & $1(20 \%)$ & $2(40 \%)$ & $4(80 \%)$ & $0(0 \%)$ \\
\hline Nitrofurantoin & $102(25.9 \%)$ & $38(56.7 \%)$ & $5(100 \%)$ & $4(80 \%)$ & $4(80 \%)$ & $2(50 \%)$ \\
\hline Cefotaxime & $295(74.9 \%)$ & $43(64.2 \%)$ & $2(40 \%)$ & $2(40 \%)$ & $4(80 \%)$ & $4(100 \%)$ \\
\hline
\end{tabular}

\section{DISCUSSION}

The present study focused on distributions of uropathogens in confirmed UTI cases from in-patients and out-patients. In our study, most of the samples were received from males than females which is contrary to locally and internationally available literature [2, 16-18] The current study was conducted in SIUT which is a kidney transplant center. Most of the admitted patients comprise those requiring a kidney transplant or those who present with a major complaint of other kidneyrelated issues in which UTI is common. Secondly, the male gender was predominant among admitted patients during the study period. Moreover, the majority of the specimen included in the study were those collected from the in-patient department, which could be the most likely explanation for higher UTI samples from the male population in our study.

The bacterial spectrum in UTI of hospitalized patients is extensive, including Enterobacteria, non-fermenters and Gram-positive bacteria. However, the bacterial spectrum of pathogens from the urinary tract causing septicaemia is markedly shifted toward Gram-negative organisms [19, 20]. In our study, Gram-negative bacteria were isolated from $99.2 \%$ of UTI cases. A similar study was conducted in Punjab, Pakistan to ascertain the spectrum of isolates from UTI cases and their resistance pattern against commonly used antibiotics. The study reported that $70.2 \%$ of UTI cases were caused by Gram-negative bacteria [16]. The finding of a higher frequency of gram-negative bacteria is also in line with other study conducted in Pakistan [18]. The findings regarding the higher frequency of Gram-negative bacteria are also consistent with the studies conducted in Iran and Ethiopia [21, 22].
In the present study, the overall most frequent isolated microorganism was E-coli (82.1\%) followed by Klebsiella spp (14\%), Pesudomonas Aer (1\%), Proteus Mir (1\%), Morganella (1\%) and Staph Aureus (0.8\%). The findings of the current study were similar to another Pakistani study in terms of the most frequently detected organism as E.coli was also the most detected organism in that study. However, findings contrasted regarding Gram-positive bacteria as in our study the only detected Gram-positive bacteria was Staph aureus whereas in that study Enterococcus faecalis (14.8\%) and Candida (14.3\%) were also detected in $14.8 \%$ and $14.3 \%$ of the UTI cases which stood as second and third frequently detected organism and other detected organisms were Pseudomonas (5.9\%), Klebsiella spp. (1.3\%), Staphylococcus aureus (0.8\%), Proteus (0.8\%) [16]. Hrabcek et al. conducted a study in Central Europe and observed that the most frequently isolated bacteria was E.coli $(26.0 \%)$, followed by Enterococcus spp. (22.4\%), Klebsiella spp. (11.3\%), P. aeruginosa (7.3\%), and Proteus spp. (6.2\%). Hrabcek et al. in their study also reported that the relative prevalence of microorganisms was varying with the time with an increase in the prevalence of E. coli and Proteus spp [17].

In the present study, we also determined the antibiotic susceptible pattern of detected uropathogens for tailoring the empirical antibiotic treatment according to the local epidemiological condition. A high resistance of E.coli was observed to cefotaxime $(74.9 \%)$, ceftazidime (74.6\%), cefoxitin (70.1\%) and augmentin 63.2\%). Lesser resistance rate of E.coli was observed for imipenem (7.4\%), fostomycin (8.9\%), amikacin (13.2\%) and nitrofurantoin $(25.9 \%)$. Sohail et al. in their study reported approximately the same resistance rate of E.coli to cefotaxime $(72 \%)$, Ceftazidime $(71 \%)$ however 
resistance to Amoxicillin (84\%) was quite higher than observed in our study. Similarly, Sohail et al. reported lower resistance of E.coli to imipenem (3\%), fosfomycin $(10 \%)$. In contrast to our study, high resistance of E.coli was observed to amikacin (91\%). However, he did not test E.coli for nitrofurantoin and cefoxitin [18]. A study conducted in 2008 in Pakistan reported resistance pattern of E.coli for the following antibiotics; ampicillin (92\%), co-trimoxazole (80\%), ciprofloxacin (62\%), gentamicin $(47 \%)$, nitrofurantoin $(20 \%)$, and amikacin (4\%) [23].

The second most isolated organism in our study was Klebsiella spp which had the highest resistance for cefoxitin $(73.1 \%)$, cefotaxime $(64.2 \%)$, ceftazidime $(64.2 \%)$, augmentin $(55.2 \%)$, nitrofurantoin $(56.7 \%)$ and lower resistance was observed for imipenem (13.4\%), fosfomycin (17.9\%), and amikacin $(28.4 \%)$. The lowest resistance of Klebsiella spp was observed against amikacin (37\%) imipenem (37\%) in other Pakistani investigation while ceftazidime $(85.2 \%)$, cefotaxime $(100 \%)$, nitrofurantoin $(85.2 \%)$ were highly resistant antimicrobials against Klebsiella spp. In this study, a much higher resistance was observed for fosfomycin than our findings (48\%) [18].

In the present study, Pseudomonas spp was found to be $100 \%$ resistant to cefoxitin and nitrofurantoin. High resistance was also observed for augmentin (60\%), imipenem (60\%), cefotaxime $(40 \%)$ and ceftazidime (40\%) whereas it was $100 \%$ and $80 \%$ sensitive for amikacin and fosfomycin respectively. A lower resistance for amikacin was also observed in a study conducted by Sohail et al. and resistance to all other antibiotics was not tested in his study [16]. A study conducted in Khyber Pakhtun Khuwa reported 8.3\% resistance to amkacin, $25 \%$ resistance to augmentin, $66.7 \%$ resistance to cefotaxime, $75 \%$ resistance to ceftazidime, $58.3 \%$ resistance to fosfomycin, $41.7 \%$ resistance to imipenem and $100 \%$ resistance to nitrofurantoin. Resistance to cefoxitin was also not tested in this study [18].

We found Proteus mir. was highly resistant against augmentin $(100 \%)$ followed by nitrofurantoin $(80 \%)$, cefoxitin (60\%), amikacin (40\%), fosfomycin (40\%), cefotaxime $(40 \%)$, ceftazidime $(40 \%)$ whereas highly sensitive to imipenem (100\%). These findings are consistent with the study conducted by Muhmmad et al. who reported similar resistance of Proteus mir. against nitrofurantoin $(83.3 \%)$, cefoxitin $(66.7 \%)$, fosfomycin (33\%). However, contradictory to our study, higher resistance was observed for amikacin (66.7\%), ceftazidime $(50 \%)$ and imipenem $(16.7 \%)$ whereas augmentin was not tested in his study for Proteus mir. [18]. A resistance of $67 \%$ was observed for amoxicillin in a study by Sohail et al. [16].

Studies conducted in Pakistan, Kuwait and Central Europe did not observe M. morganii isolate in their study [2, 16-18]. However, a case report was published from Iran in which they reported that for a first time they observed M. morganii isolate harboring blaVIM, blaCTX-M, and blaSHV genes after kidney transplantation with persistent urinary infections. The current study was conducted in SIUT which is a Kidney transplant center more probably, unlike other studies, we also found the $\mathrm{M}$. moraganii as a rare cause of UTI due to this fact. However, future research should be conducted with a larger sample size to further have the evidence. M. morganii is naturally resistant to tetracyclines, tigecycline, polymyxins, and nitrofurantoin [24]. Consistent with the literature, in our study, we also found $80 \%$ resistance of M. morganii against nitrofurantoin.

In our study, although microorganism Staph auerus was found in $0.83 \%$ cases of UTI however, it was $100 \%$ resistant to augmentin, cefoxitin, cefotaxime, ceftazidime. Resistance of $50 \%$ was also observed for amikacin and nitrofurantoin with high sensitivity for fosfomycin (100\%) and imipenem (25\%). In contrast to our study, a resistance of $84.6 \%, 85.7 \%, 42.9 \%, 50 \%, 14.3 \%$ was observed against augmentin, cefotaxime, ceftazidime, amikacin, fosfomycin and imipenem respectively. Resistance against cefoxitin was not determined in other studies [18].

\section{CONCLUSION}

The present study demonstrated that gram-negative bacteria was the most frequent cause of urinary tract infection. Microorganisms showed variable resistance to different antibiotics. The first line of antibiotics should be rationally selected by physicians to treat urinary tract infections.

\section{ETHICS APPROVAL}

The study was conducted after obtaining approval from Hospital Ethics Committee. 


\section{CONSENT FOR PUBLICATION}

Not applicable.

\section{FUNDING}

None.

\section{CONFLICT OF INTEREST}

The authors declare no conflict of interest.

\section{ACKNOWLEDGEMENTS}

We acknowledged all the microbiology staff of Sindh Institute of Urology and Transplantation who facilitated who were performing testing of the specimens and reporting the final status.

\section{REFERENCES}

1. Tan CW, Chlebicki MP. Urinary tract infections in adults. Singapore Med J 2016; 57(9): 485-90.

2. Al Sweih N, Jamal W, Rotimi V. Spectrum and antibiotic resistance of uropathogens isolated from hospital and community patients with urinary tract infections in two large hospitals in Kuwait. Med Princ Pract 2005; 14(6): 401-7.

3. Zowawi HM, Harris PN, Roberts MJ, Tambyah PA, Schembri MA, Pezzani MD, et al. The emerging threat of multidrug-resistant Gram-negative bacteria in urology. Nat Rev Urol 2015; 12(10): 570.

4. Totsika M, Gomes MD, Idris A, Rogers AB, Wurpel JD, Phan M-D, et al. Uropathogenic Escherichia coli mediated urinary tract infection. Curr Drug Targets 2012; 13(11): 1386-99.

5. Stamm WE, Norrby SR. Urinary tract infections: disease panorama and challenges. J Infect Dis 2001; 183(Supplement_1): S1-S4.

6. Barber AE, Norton JP, Spivak AM, Mulvey MA. Urinary tract infections: current and emerging management strategies. Clin Infect Dis 2013; 57(5): 719-24.

7. Schaeffer A, Rajan N, Cao Q, Anderson B, Pruden DL, Sensibar J, et al. Host pathogenesis in urinary tract infections. Int J Antimicriob Agents 2001; 17(4): 245-51.

8. Tabibian JH, Gornbein J, Heidari A, Dien SL, Lau VH, Chahal P, et al. Uropathogens and host characteristics. J Clin Microbiol 2008; 46(12): 3980-6.

9. Katouli M. Population structure of gut Escherichia coli and its role in development of extra-intestinal infections. Iran J Microbiol 2010; 2(2): 59.
10. Ronald A. The etiology of urinary tract infection: traditional and emerging pathogens. Am J Med 2002; 113(1): 14-9.

11. Subramanian M, Ganesapandian S, Singh M, Kumaraguru A. Antimicrobial susceptibility pattern of urinary tract infection causing human pathogenic bacteria. Asian J Med Sci 2011; 3(2): 56-60.

12. Ciorba V, Odone A, Veronesi L, Pasquarella C, Signorelli C Antibiotic resistance as a major public health concern: epidemiology and economic impact. Ann Ig 2015; 27(3): 562-79.

13. Kass EH. Asymptomatic infections of the urinary tract. Trans Assoc Am Phys 1956; 69: 56-64.

14. Barrow GI, Feltham RKA. Cowan and steel's manual for the identification of medical bacteria. $3^{\text {rd }}$ ed. New York: Cambridge University Press 1993.

15. Clinical and Laboratory Standards Institute. performance standards for antimicrobial susceptibility testing. $28^{\text {th }}$ ed. CLSI supplement M100. Wayne, PA: Clinical and Laboratory Standards Institute 2018.

16. Sohail M, Khurshid M, Saleem HG, Javed $H$, Khan AA. Characteristics and antibiotic resistance of urinary tract pathogens isolated from Punjab, Pakistan. Jundishapur J Microbiol 2015; 8(7): e19272.

17. Hrbacek J, Cermak P, Zachoval R. Current antibiotic resistance trends of uropathogens in Central Europe: Survey from a Tertiary hospital urology department 2011-2019. Antibiotics 2020; 9(9): 630.

18. Muhammad A, Khan S, Ali N, Rehman M, Ali I. Prevalence and antibiotic susceptibility pattern of uropathogens in outpatients at a tertiary care hospital. New Microbes New Infect 2020; 36: 100716.

19. Kibret M, Abera B. Antimicrobial susceptibility patterns of $E$. coli from clinical sources in northeast Ethiopia. African Health Sci 2011; $11: 40-5$

20. Sabir S, Anjum AA, ljaz T, Ali MA. Isolation and antibiotic susceptibility of $E$. coli from urinary tract infections in a tertiary care hospital. Pak J Med Sci 2014; 30(2): 389.

21. Seifu WD, Gebissa AD. Prevalence and antibiotic susceptibility of Uropathogens from cases of urinary tract infections (UTI) in Shashemene referral hospital, Ethiopia. BMC Infect Dis 2018; 18(1): 1-9.

22. Mirzarazi M, Rezatofighi SE, Pourmahdi M, Mohajeri MR. Antibiotic resistance of isolated gram negative bacteria from urinary tract infections (UTIs) in Isfahan. Jundishapur J Microbiol 2013; 6(8): e6883

23. Bashir MF, Qazi JI, Ahmad N, Riaz S. Diversity of urinary tract pathogens and drug resistant isolates of Escherichia coli in different age and gender groups of Pakistanis. Trop J Pharm Res 2008; 7(3): 1025-31.

24. Leclercq R, Cantón R, Brown DF, Giske CG, Heisig P, MacGowan AP, et al. EUCAST expert rules in antimicrobial susceptibility testing. Clin Microbiol Infect 2013; 19(2): 141-60. 